\title{
Factors Influencing the QMF Resolution for Operation in Stability Zones 1 and 3
}

\author{
Sarfaraz U. A. H. Syed, Thomas Hogan, John Gibson, Stephen Taylor \\ Department of Electrical Engineering and Electronics, University of Liverpool, Liverpool, L69 3GJ, UK
}

\begin{abstract}
This study uses a computer model to simulate a quadrupole mass filter (QMF) instrument under different operating conditions for Mathieu stability zones 1 and 3 . The investigation considers the factors that limit the maximum resolution $\left(R_{\max }\right)$, which can be obtained for a given QMF for a particular value of scan line. Previously, QMF resolution $(R)$ has been found to be dependent on number $(\mathrm{N})$ of radio frequency ( $\mathrm{rf}$ ) cycles experienced by the ions in the mass filter, according to $\mathrm{R}=N^{n} / K$, where $n$ and $K$ are the constants. However, this expression does not predict the limit to QMF resolution observed in practice and is true only for the linear regions of the performance curve for QMF operation in zone 1 and zone 3 of the stability diagram. Here we model the saturated regions of the performance curve for QMF operation in zone 1 according to $R=q\left(1-2 c^{N}\right) / \Delta q$, where c is a constant and $\Delta q$ is the width of the intersection of the operating scan line with the stability zone 1 , measured at $q$-axis of the Mathieu stability diagram. Also by careful calculations of the detail of the stability tip of zone 1, the following relationship was established between $\mathrm{R}_{\max }$ and percentage $U / V$ ratio: $R_{\max }=q /(0.9330-0.00933 U / V)$. For QMF operation in zone 3 the expression $R=a-b c^{N}$ simulates well the linear and saturated regions of the performance curve for a range of operational conditions, where $\mathrm{a}, \mathrm{b}$, and $\mathrm{c}$ are constants.
\end{abstract}

Key words: Quadrupole mass filter (QMF), Resolution, U/V ratio, Number of RF cycles, Performance curve, Runge-Kutta algorithm

\section{Introduction}

A quadrupole mass spectrometer (QMS) is an instrument that is widely used for the identification of charged particles according to their mass to charge $(\mathrm{m} / \mathrm{z})$ ratio. The QMS contains three elements; (1) ion source, (2) quadrupole mass filter (QMF), and (3) ion detector [1]. The wide range of applications involving QMS in both industry and research give an impressive testimony to the value of this instrument $[2,3]$. More recently, portable QMS have been used in harsh environments for oil-in-water monitoring and analysis [4]. Different applications have different requirements of resolution, sensitivity, and stability of operation which depend in turn on the QMS operating conditions.

The history of QMF dates back to the pioneering work of Wolfgang Paul and co-workers in the 1950s [5]; and since

Correspondence to: Stephen Taylor; e-mail: s.taylor@liv.ac.uk then much research has been undertaken by different researchers to study the performance and behavior of the QMF by either experimental methods or by numerical simulation techniques. In 1962, Von Zahn, a member of the Paul group, constructed a QMF of $5.82 \mathrm{~m}$ in length and reported a resolution of the order of 16,000 , for highprecision mass measurements [6]. Brubaker and Tull investigated the transmission efficiency and resolution of a QMF as a function of QMF ion source aperture size and frequency of excitation; the ion source exit aperture defines the area of illumination of the QMF and is usually a fraction of the inscribed QMF field radius $\left(r_{0}\right)$ [7]. It was concluded that higher resolution is obtained with small aperture size and higher frequency. Holme et al. performed a series of experiments to investigate the factors that determine the maximum resolution of a QMF under normal operating conditions [8]. Later, Dylla and Jarrell showed experimentally that by using an entrance aperture that spatially separates the rf and dc fringe fields in a QMF, transmis-

Received: 15 September 2011

Revised: 18 January 2012

Accepted: 24 January 2012

Published online: 11 February 2012 
sion-resolution characteristics can be improved [9], an idea that was originally proposed by Brubaker [10]. In Dawson (1976), the design and performance of the QMF is described in detail, and it was reported that resolution $\mathrm{R}$ can be represented by $R=N^{n} / K$, where $n$ is either exactly or closely equal to 2, and $K$ is a constant that may be assumed to be 20 for all practical purposes [1, chapter 6].

Since then, there have been many analytical predictions of QMF behavior. Batey showed that a number of features of the behavior of the QMF could be predicted by tracing ion trajectories through the QMF [11]. Muntean developed a computer simulation program using matrix methods to study the transmission through the QMF by calculating ion trajectories in rf only quadrupoles [12]. Some workers have modeled ion transmission through the filter by calculating ion trajectories in exactly determined quadrupole fields [13]. Other workers have used computed spectra to investigate zone 1 performance under a number of operating conditions; their computer program generates large number of ions (at least $10^{5}$ ions injected into the quadrupole model at each point on the mass scale), thus providing a detailed computer simulation for both hyperbolic and round-electrodes [14, 15]. Du et al. have performed elemental analysis with QMF operated in higher stability regions, and it was observed that there is an increase in resolution when QMF is operated in higher stability regions [16]. Hogan and Taylor performed computer simulation for a QMF operating in first and third stability zones, and it was concluded that operation in zone 3 provides an improved immunity from the effects of the variation in the value of electrode radius $r$ to field radius $r_{0}$ (referred as $r / r_{0}$ ) compared with zone 1 [17].

\section{Theory}

A QMF consisting of a parallel array of four electrodes mounted in square configuration produces a central electric field whose strength increases linearly with increasing displacement from the central axis and is independent in $x$ and $y$ (ideal quadrupole field). When driven by the correct combination of dc and rf voltages, the electrode assembly is capable of providing mass filtering action. With the voltages defined in equations 1 and 2, the behavior of the ions as they pass through the QMF can be defined by the Mathieu Equation 3 [18].

$$
\begin{gathered}
\phi_{x}=(U-V \cos \omega t) \\
\phi_{y}=-(U-V \cos \omega t) \\
\frac{d^{2} u}{d \xi^{2}}+\left(a_{u}-2 q_{u} \cos (2 \xi)\right) u=0
\end{gathered}
$$

where $u=x$ or $y$ and

$$
\begin{aligned}
& a_{u}=a_{x}=-a_{y}=\left(\frac{8 e U}{m r_{0}^{2} \omega^{2}}\right) \\
& q_{u}=q_{x}=-q_{y}=\left(\frac{-4 e V}{m r_{0}^{2} \omega^{2}}\right)
\end{aligned}
$$

where $V$ is the zero-to-peak amplitude of rf voltage oscillating with angular frequency $\omega$ (expressed in radians per second), $U$ is the applied dc voltage, $t$ is the time, $e$ is the charge on the ion, and $m$ is the ion's mass. Under normal operating conditions, the mass filtering action of the QMF is controlled by electrode voltages $U$ and $V$. The ratio of the voltages $U$ and $V$ controls the QMF resolution setting and the voltage $V$ sets the mass scale. The mass scan line shows the $a$ and $q$ values of ions of different mass to charge ratios for a given $\mathrm{dc}$ and $\mathrm{rf}$ voltage ratio. QMF resolution was found to be dependent on number of $\mathrm{rf}$ cycles $(\mathrm{N})$ experienced by the ions in the mass filter. $\mathrm{N}$ may be calculated by: $N=(f l) / \sqrt{ }\left(2 E_{z} / m\right)$ where $l$ is the length of the mass filter, $f$ is frequency of the rf voltage and $E_{z}$ the ion energy. From the mass spectrum obtained by the numerical simulation, the resolution $R$ can be calculated by using the equation $R=M / \Delta M$, where $M$ is the mass of the given spectral peak and $\Delta M$ is the width of the mass peak measured at $10 \%$ of its height [1].

\section{Computer Models Used}

\section{QMS2-Hyperbolic}

A custom software program (QMS2-hyperbolic) was used to study the performance of QMF. This computes individual ion trajectories by solving the Mathieu equations using a fourth order Runge-Kutta algorithm. It operates by dividing the ion trajectories into small steps and assuming that over the steps, the ion motion in three directions $x, y$, and $z$ is uncoupled. Mass scans are computed by ramping the values of $U$ and $V$, and the $U / V$ ratio sets the resolution of an instrument. The program provides generation, display, and storage of mass spectra and individual ion trajectories, together with parametric sweep control.

A second program (IonSrc) has been used to generate the entry conditions for a large number of ions (typically $10^{8}$ ). Ions with a uniform distribution (illumination) across the field aperture and randomly placed with respect to the rf phase were used. Finally, Matlab and OriginPro 8.5 are used to postprocess the data and for the generation of graphical results. 


\section{Results and Discussion}

\section{Performance of a QMF Operating in Stability Zone 1}

All the simulations use 300 steps across the mass range with $5 \times 10^{5}$ ions injected and traced at each mass step. Mass peaks for ${ }^{40} \mathrm{Ar}^{+}$are generated for a hyperbolic quadrupole mass filter with inscribed radius (field radius) of $0.9 \mathrm{~mm}$. The frequency of the rf voltage used in the simulation was $3.686 \mathrm{MHz}$ and the input ion energy $\left(E_{z}\right)$ was chosen as $5 \mathrm{eV}$. The ion source radius $\left(r_{i e}\right)$ was selected as $0.5 \mathrm{~mm}$ and the exit radius (detector radius) was chosen as $1.8 \mathrm{~mm}$. The length of the mass filter was varied in order to vary the number of $\mathrm{rf}$ cycles the ion experiences. It is assumed in the model that the QMF control setting $U / V=100 \%$ corresponds to the peak of the stability diagram (i.e., $a=0.23699$ and $q=$ 0.706 for zone 1). No account of fringing fields at the entrance or exit of the mass filter has been considered, and the initial velocities in the $x$ and $y$ directions are zero. In practice at the entrance and exit of QMF fringe fields are present. These are regions of approximately $r_{0}$ in length both inside and outside the quadrupole where field grows from 0 to its maximum value within the quadrupole [19]. The fringe fields in this region can have a defocusing effect upon ions. For example if the $U / V$ ratio is high and if the fringe field grows linearly, ions may be in a state of unstable motion and may be rejected before they reach the QMF. Figure 1a shows the dependence of resolution on number of $\mathrm{rf}$ cycles at $U / V$ ratios $99.9 \%, 99.95 \%, 99.99 \%$, and $99.998 \%$. For the linear region of each curve the results satisfy the previously reported experimental relationship between resolution and number of $\mathrm{rf}$ cycles [1], $\mathrm{R}=N^{n} / K$, and the values of $n$ and $K$ at different $U / V$ ratios obtained via OriginPro 8.5 (curve fitting software) are shown in Table 1. As observed from Table 1 , the values of $n$ and $K$ are in accord with values previously determined for a range of experimental measurements [1, chapter 6] with the precise values of $n$ and $K$ dependent upon the value of $U / V$ ratio. It can be seen from Figure 1a that in all cases, as the number of rf cycles increases, resolution increases with a finite slope and then saturates. To test whether this was a true result or an artifact of the program, the same instrumental conditions were simulated using different computing parameters. However, even after decreasing the mass range, increasing the number of steps up to 1200 , and the number of ions to $1 \times 10^{8}$, and integrating with fine settings (i.e., small integration intervals, so as to increase the Runge-Kutta time step size), the same results were obtained. It was concluded that results were not due to the computer model parameters or the method, but rather represented a feature of ion motion in an ideal quadrupole field. Figure $1 \mathrm{~b}$ shows a computed mass spectrum for ${ }^{40} \mathrm{Ar}^{+}, \mathrm{M}$ is the mass of the given spectral peak and $\Delta M$ is the width of the mass peak measured at $10 \%$ of its height.

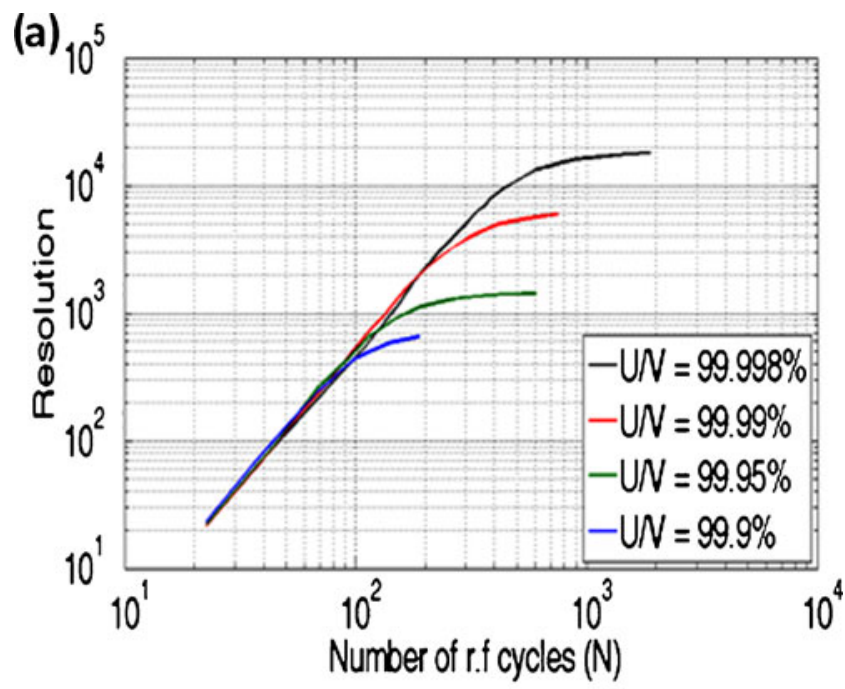

(b)

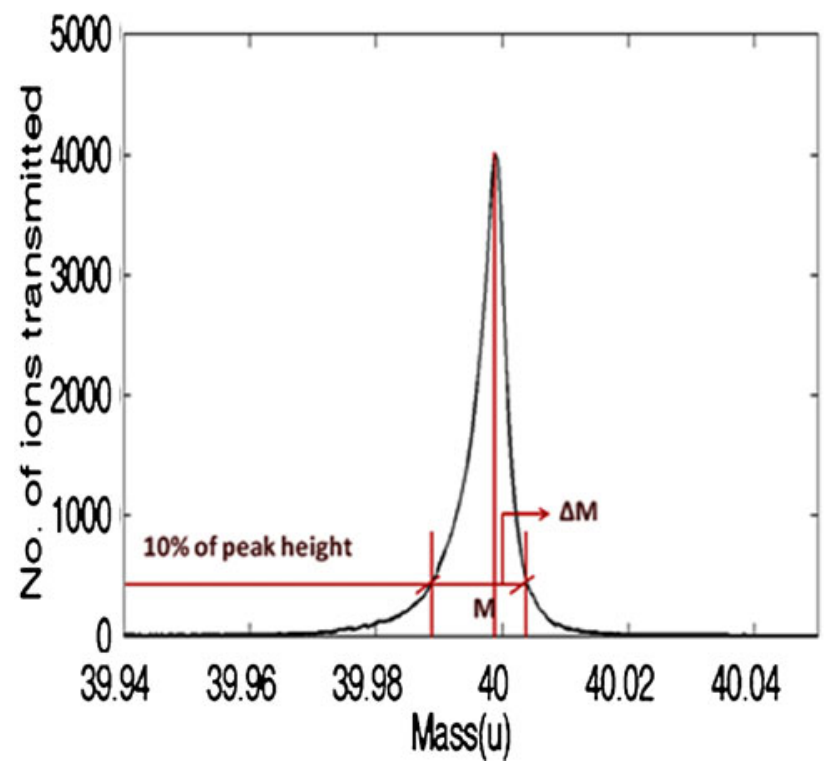

Figure 1. (a) The dependence of resolution on the number of if cycles for stability zone 1 at different $U / V$ ratios; predicted by QMS2-hyperbolic; (b) a model of a mass spectrum for ${ }^{40} \mathrm{Ar}^{+}$

For $U / V<100 \%$, it can be concluded that there is finite theoretical limit to resolution for a particular value of instrument operating point set by the $U / V$ ratio (scan line),

Table 1. Values of $n$ and $K$ at Different $U / V$ Ratios (Zone 1 Operation)

\begin{tabular}{lll}
\hline$U / V$ ratio & $n$ & \multicolumn{1}{c}{$K$} \\
\hline $99.9 \%$ & 1.954 & 16.155 \\
$99.95 \%$ & 1.954 & 15.84 \\
$99.99 \%$ & 2.02 & 20.981 \\
$99.998 \%$ & 2.08 & 28.02 \\
\hline
\end{tabular}


as at that particular point $\Delta q$ cannot go beyond a certain minimum obtainable value. This is because the intersection of the scan line with the stability diagram will give a minimum obtainable value of $\Delta q$ and correspondingly $\Delta M$. The Mathieu stability diagram for zone 1 with the intersection of the scan line at two operating points is shown in Figure 2. Figure 2a shows the Mathieu stability diagram for zone 1 with the intersection of the scan line at $100 \%$; whereas Figure $2 \mathrm{~b}$ shows the intersection at $U / V<100 \%$. At

(a)

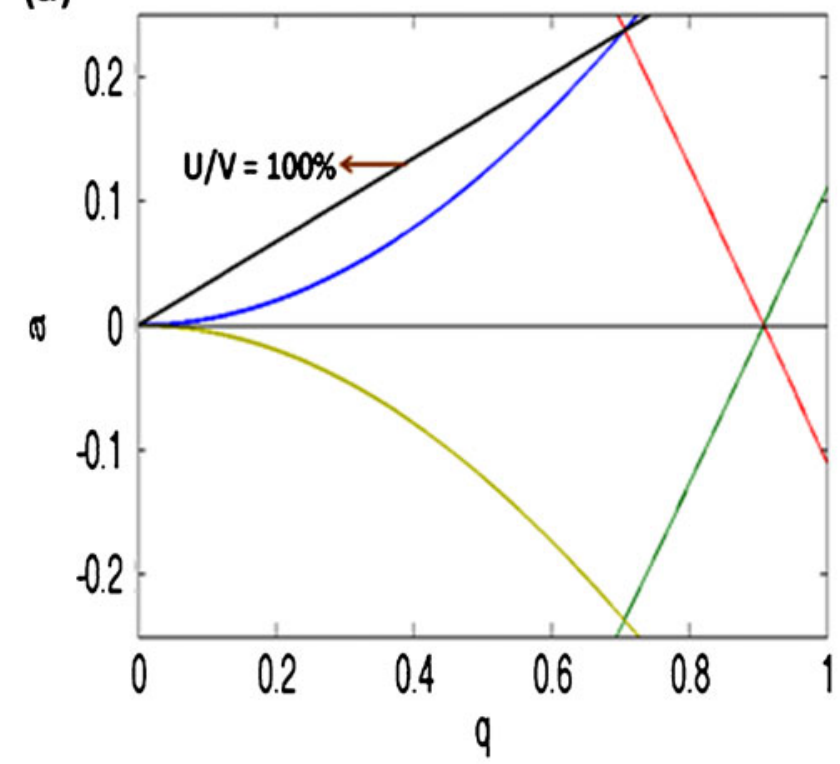

(b)

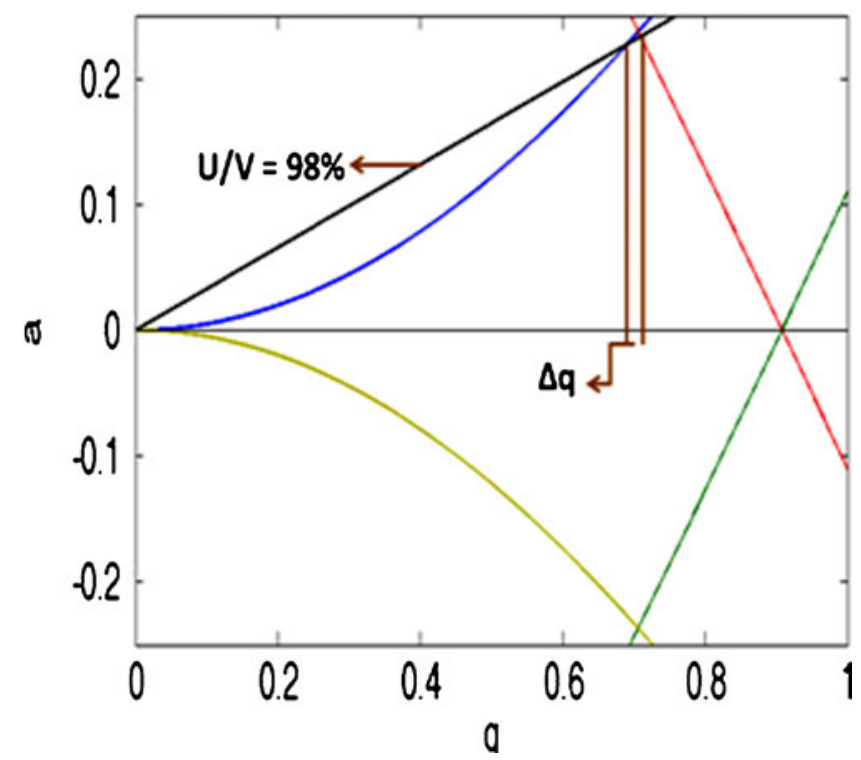

Figure 2. (a) The Mathieu stability diagram for zone 1 with the intersection of the scan line at $100 \%$; (b) the Mathieu stability diagram for zone 1 with the intersection of the scan line at $U / V<100 \%$
$100 \% \Delta q$ should be ideally zero; whereas, at $U / V<100 \% \Delta q$ has a certain finite non zero value. Intersection of the scan line below the tip (Figure 2b) increases the pass-band and in turn limits the resolution of a QMF to a finite maximum value, which is given by $\left(R_{\max }=q / \Delta q=0.706 / \Delta q\right)$ for a given mass even if the number of rf cycles are increased by increasing the length or frequency or by decreasing ion energy.

For the saturation regions of the performance curves, observed in practice [1] and modeled in Figure 1a, a good representation is given by a function of the form $(R=a-$ $b c^{N}$ ), where $\mathrm{a}, \mathrm{b}$, and $\mathrm{c}$ are constants. Figure $3 \mathrm{a}$ (square symbols) shows the relationship between $N$ and $R$ obtained using QMS2-hyperbolic and a fitted curve to the data (red line) using OriginPro 8.5 for $U / V$ ratio $99.99 \%$. The value of the coefficient of determination (COD) termed as $R_{c}{ }^{2}$ was

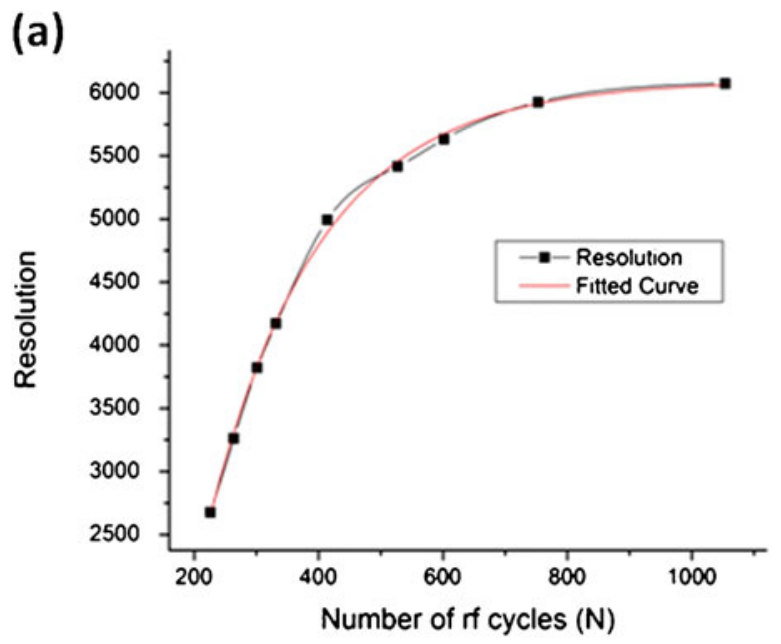

(b)

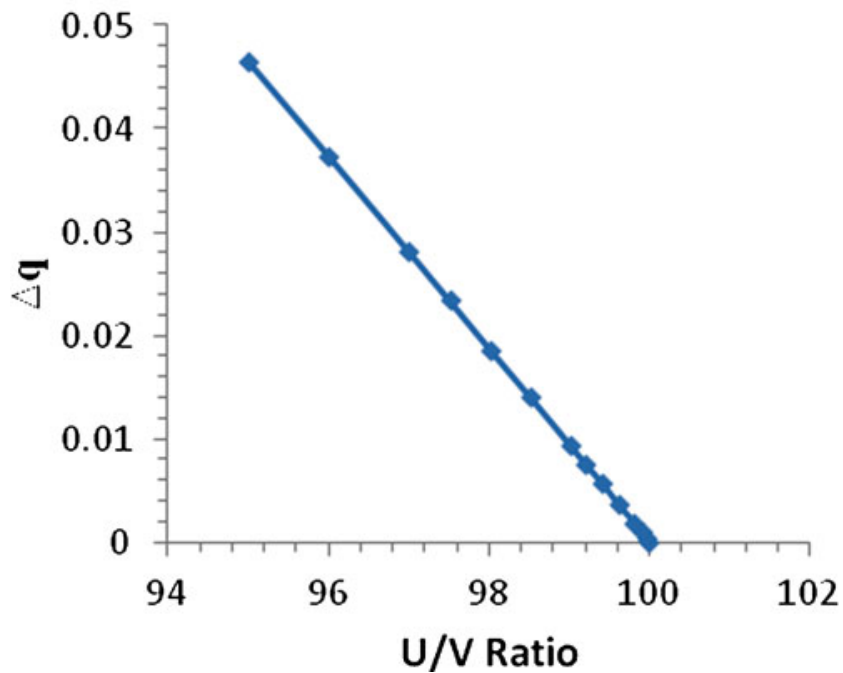

Figure 3. (a) The dependence of resolution on number of if cycles for the saturation region of the performance curve for stability zone 1 at $U / V=99.99 \%$ (fit obtained using Origin 8.5); (b) variation of $\Delta q$ with $U / V$ ratio for stability zone 1 
found to be 0.9985 , indicating a good regression fit between the curves $\left(R_{c}{ }^{2}\right.$ of 1 indicates that the regression line perfectly fits the data). The value of adj. $R_{c}{ }^{2}$ (a modification of $R_{c}{ }^{2}$ that adjusts for the number of independent terms in a model) was found to be 0.9981 . The values of the parameters $a, b$, and $c$ depend upon the $U / V$ ratio used in the QMS2-hyperbolic simulations. The same approach can be applied to model all the curves of Figure 1a and the corresponding Table 2 shows the values of the parameters $a$, $b, c$, and the coefficient of determination $R_{c}{ }^{2}$ at different $U / V$ ratios.

From the values obtained, it is observed in all cases that the parameters $a$ and $b$ have a constant ratio of approximately 0.49 . Moreover the value of parameter $a$ is found to be approximately $q / \Delta q$, except for $U / V=99.998 \%$, and this discrepancy will be explained later in the paper. By substituting these observations in the functional relationship determined from the curve fit, and for simplicity considering the ratio of $a / b$ as 0.5 , for the saturation region of the performance curve, $R$ can be related to $N$ using $R=q(1-$ $\left.2 c^{N}\right) / \Delta q$. It was previously thought that the saturation in the performance curve was as a result of imperfections in QMF manufacturing [1]. Our simulations using QMS2 hyperbolic are for the case of an ideal (but finite length and finite field radius) quadrupole field with zero mechanical misalignments. The saturation behavior in resolution observed by Holme et al. [8] with increasing $N$ is thus seen to be the feature of the QMF and therefore not necessarily due to mechanical deficiencies in QMF manufacture.

\section{Dependence of Ultimate Maximum Resolution on U/V Ratio}

To calculate the details of the stability tip, a program that is a direct conversion of the corresponding Fortran program in Zhang and Jin [20], was used. The program generates the boundaries of the stable and unstable areas of the Mathieu stability diagram in a discretized form. These values are then imported into a Matlab program, which plots the full Mathieu stability diagram. The scan line is then drawn over the Mathieu equation and the points of intersection with the Mathieu stability boundaries can be detected. By altering the active area of the graph and the scan line, different scan line stability zone combinations can be plotted. By careful

Table 2. Values of Parameters a, b, c, and CODs for QMF Operation in Zone 1

\begin{tabular}{lccll}
\hline Variables & $U / V 99.9 \%$ & $U / V 99.95 \%$ & $U / V 99.99 \%$ & $U / V 99.998 \%$ \\
\hline a & 725.80 & 1425.35 & 6093 & 17946 \\
b & 1516.28 & 2882.27 & 12237.96 & 36598.97 \\
c & 0.98278 & 0.98846 & 0.99441 & 0.99666 \\
COD- $R_{c}{ }^{2}$ & 0.9994 & 0.999 & 0.9985 & 0.9977 \\
Adj. $R_{c}{ }^{2}$ & 0.9991 & 0.9987 & 0.9981 & 0.9974 \\
a/b Ratio & 0.48 & 0.495 & 0.493 & 0.49 \\
\hline
\end{tabular}

calculations of the details of the stability tip, the dependency between $\Delta q$ and scan line ( $U / V$ ratio) is obtained as shown in Figure $3 \mathrm{~b}$. As expected, $\Delta q$ decreases linearly with increasing $U / V$ ratio. A good mathematical relationship between $\Delta q$ and $U / V$ ratio can be represented as $\Delta q=0.933-0.00933 U / V$. By substituting the value of $\Delta q$ in $R_{\max }=q / \Delta q$, a relationship between the maximum resolution $R_{\max }$ for a particular scan line ( $U / V$ ratio in percentage) is established and given by: $R_{\max }=q /(0.9330-0.00933 U / V)$. Figure 4a shows the comparison of maximum resolution obtained from the simulations from the saturation region of performance curve (Figure 1a) and the $R_{\max }$ calculated for the ideal case using the Mathieu stability diagram at different $U / V$ ratios. From Figure 4a the maximum resolution increases with increasing $U / V$ ratio, and as scan line approaches the tip $(U / V=100 \%)$, it starts to increase dramatically towards the singularity at $100 \%$. Moreover, it can be seen from Figure 4a, as the $U / V$ ratio increases, the discrepancy between maximum resolution obtained from simulations (i.e., the saturation
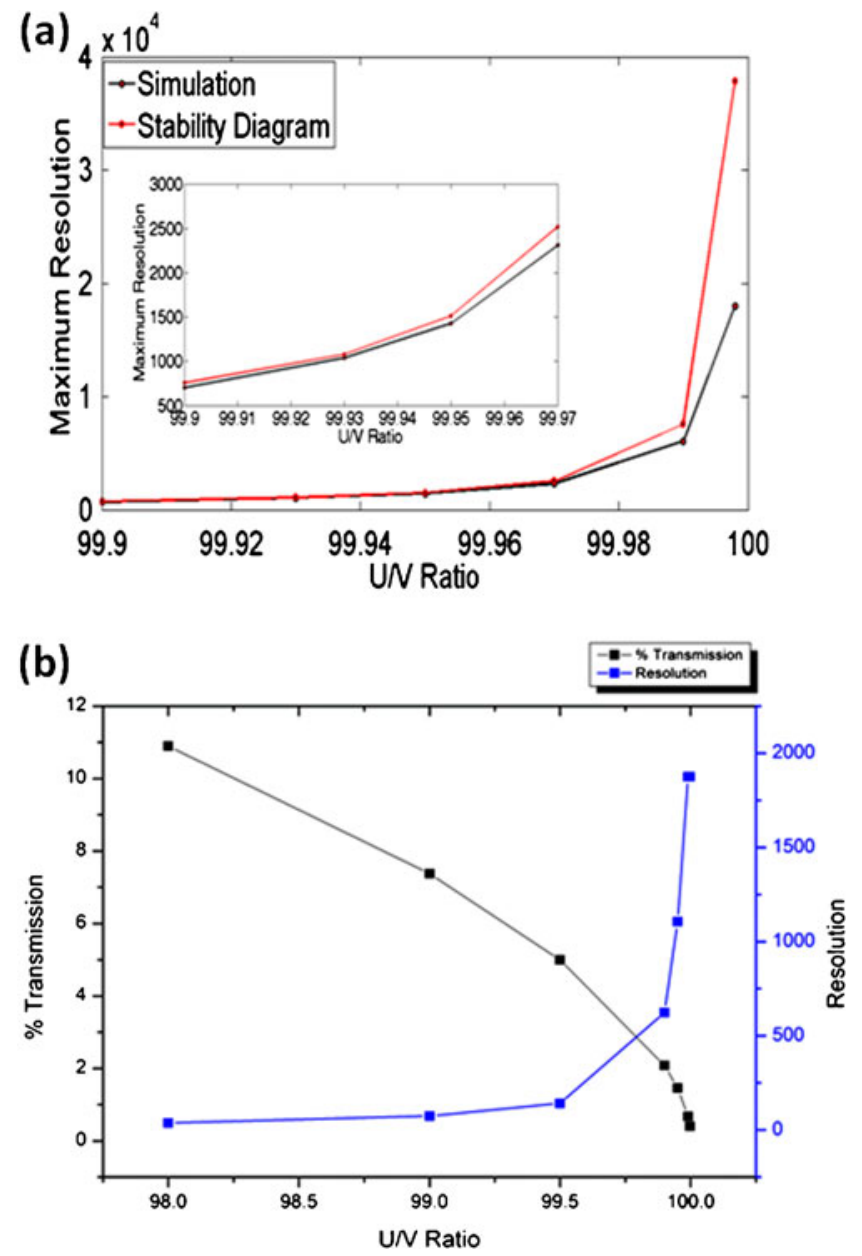

Figure 4. (a) Comparison of maximum resolution obtained in the simulations and the $R_{\max }$ calculated for the ideal case using the Mathieu Stability diagram at different $U / V$ ratios, inset $R_{\max }$ Vs $U / V$ ratio from (99.9\%-99.97\%); (b) the variation of resolution and percentage transmission with $U / V$ ratio for stability zone 1 
region of the performance curve) and from the calculations of the idealized case (stability diagram) increases. The discrepancy between maximum resolution obtained from the saturation region of the performance curve and from the stability diagram at low values of $U / V$ ratios $(99.9 \%-99.97 \%)$ is shown in the inset of Figure 4a. The discrepancy is low at lower $U / V$ ratios. The large discrepancy at high values of $U / V$ ratios can be explained with reference to Figure $2 b$ and Figure $1 b$. Figure $2 b$ also shows how the maximum resolution for a particular value of scan line is determined from the Mathieu stability diagram $(q / \Delta q)$; whereas, Figure $1 \mathrm{~b}$ shows the mass spectrum obtained by the numerical simulation and how the resolution $R$ can be calculated by using the equation $R=M / \Delta M$, where $\Delta M$ is the width of the mass peak measured at $10 \%$ of its height. Ideally (i.e., from stability diagram considerations) the shape of a mass spectral peak should be trapezoidal. However the simulation is for finite field radius and finite length and there are differences since:

1) Some ions, which are theoretically unstable, are transmitted in the simulation because of the slow increase in the ion trajectory envelope with distance along the QMF.

2) Similarly, some ions, which are theoretically stable, are lost because the ion trajectory envelope is so high that they collide with the electrode if the QMF has finite field radius.

However, in all cases, the trend observed is similar to idealized model, and only at high $U / V$ ratios there is a large discrepancy in the maximum resolution, since a slight change in $\Delta M$ or $\Delta q$ will change the resolution by a large amount. In practice $R_{\max }$ will be determined by the accuracy (percentage tolerances) of the QMF driving voltages at this point. State-of-the-art electronics to date can achieve percentage tolerances between $\pm 0.1 \%$ and $\pm 0.5 \%$ for the $\mathrm{rf}$ amplitude. For direct voltages, it is currently possible to achieve $\pm 0.1 \%$ or better. Other practical limiting factors for the electronics include operating conditions (i.e., temperature range etc.). Moreover, in practice, operation at maximum obtainable resolution is also determined by the minimum measurable signal current on the detector. Figure $4 \mathrm{~b}$ shows the variation of resolution and percentage transmission with $U / V$ ratio for a QMF operating at $188 \mathrm{rf}$ cycles. As seen from Figure 4b, the percentage transmission falls with increasing $U / V$ ratio and at $99.998 \%$ the percentage transmission is $0.399 \%$ of the total ions injected into the QMF.

Using the QMS2-hyperbolic, the 5.82 meters long QMF reported by Von Zhan [6] was simulated. At an operating point for stability zone 1 with a $U / V$ ratio of $99.998 \%$ and $N=415$, an instrument resolution of around 17,000 at $50 \%$ peak width definition was predicted using the QMS2 model which matches well the reported experimental results.

\section{Performance of a QMF Operating in Stability} Zone 3

Simulations of QMF performance in zone 3 were also carried out using QMS2-hyperbolic. In this case, each simulation used 150 steps across the mass range, with $9 \times 10^{5}$ ions injected and traced at each point. Here, the operating point at $100 \%$ corresponds to the $U / V$ ratio at $q=3.0$ and $a=2.8$, which is near the center of the stability zone 3 . With increasing $U / V$ ratio, the scan line approaches the upper left tip of stability zone 3. Figure $5 \mathrm{a}$ shows the dependence of maximum resolution on number of $\mathrm{rf}$ cycles at two different $U / V$ ratios, $104.81 \%$ and $104.82 \%$. It can be seen from the Figure $5 \mathrm{a}$ that as the number of rf cycles increases, resolution increases with a finite slope and then the curve showing the dependence starts to saturate. Comparing Figure 5a with Figure 1a, the behavior for

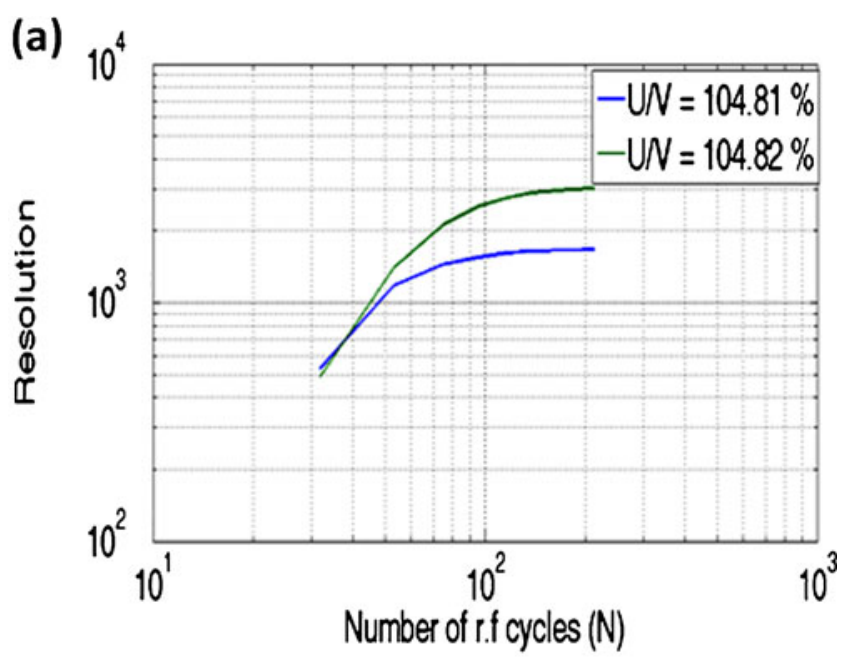

(b)

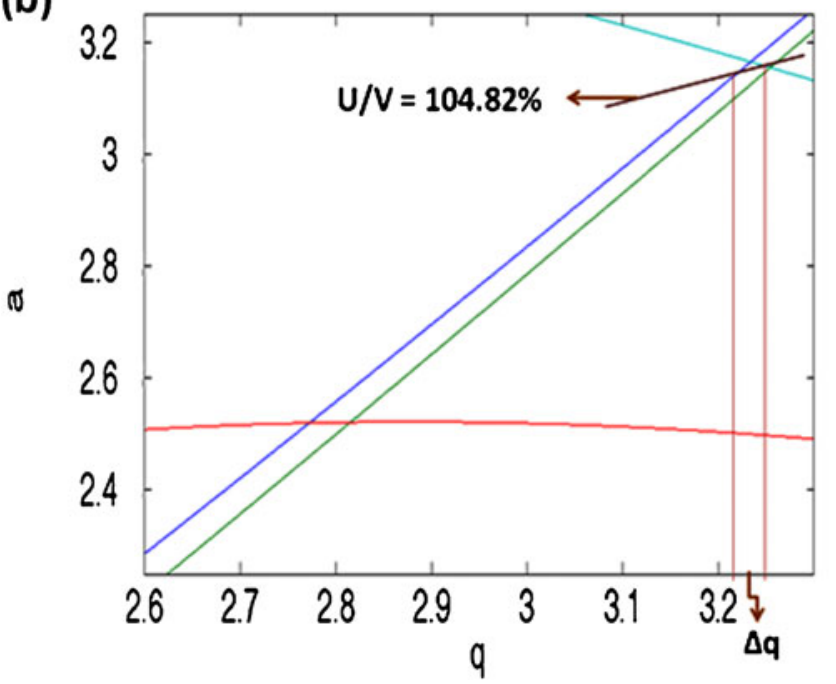

Figure 5. (a) The dependence of resolution on the number of if cycles for stability zone 3 at different $U / V$ ratios; predicted by QMS2-hyperbolic; (b) the Mathieu stability diagram for zone 3 with the intersection assumed at $U / V=$ $104.82 \%$ 


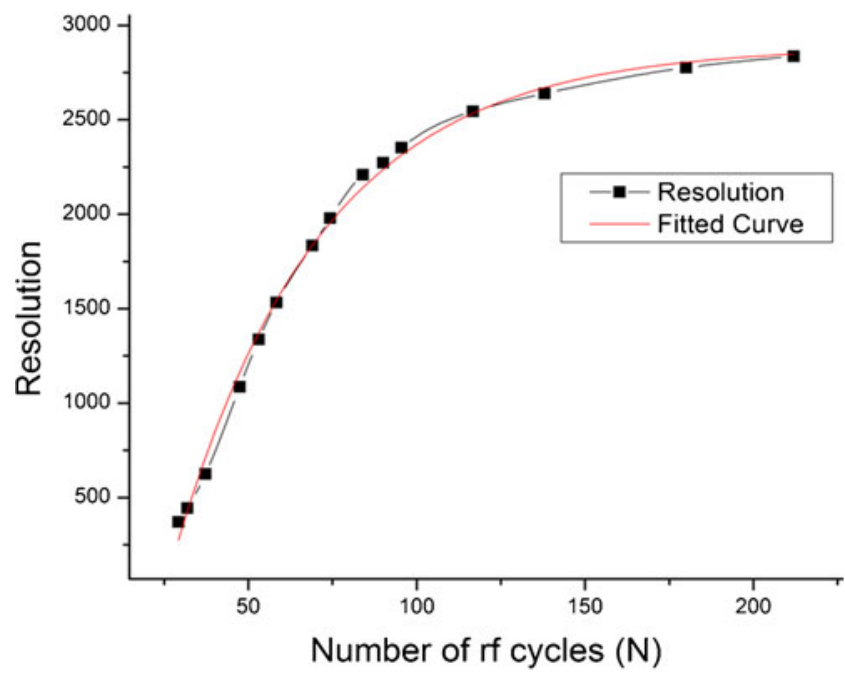

Figure 6. The dependence of resolution on number of if cycles for stability zone 3 , at $U / V=104.82 \%$ (fit obtained using Origin 8.5)

QMF operation in zone 3 is similar to that obtained in zone 1 but the predicted saturation is more pronounced in the case of zone 3 operation (i.e., occurs at a lower number of rf cycles).

For Zone 3 the values of $n$ and $K$ at $U / V$ ratio $104.82 \%$ are 2.17 and 4.22 , respectively, and for $U / V 104.81 \%$ are 1.56 and 0.43 , respectively. These values are similar to those obtained experimentally by Konenkov and Kratenko [21]. It is noticeable that for zone 3 fewer $\mathrm{rf}$ cycles are needed to achieve a given resolution compared to stability zone 1 . As seen from Figure $5 b$, the intersection of the scan line (assumed as $104.82 \%$ ) below the tip limits $\Delta q$ and in turn limits the resolution of a QMF to a maximum value for a given mass even if number of $\mathrm{rf}$ cycles are increased. Figure 6 (square symbols) shows the relationship between $N$ and $R$ for stability zone 3, obtained using QMS2-hyperbolic and a fitted curve to the data (red line) utilizing OriginPro 8.5. A good representation of the performance curve is given by $R=a-b c^{N}$, where $\mathrm{a}, \mathrm{b}$, and $\mathrm{c}$ are constants, the values of which depend upon the $U / V$ ratio. The value of the coefficient of determination $R_{c}{ }^{2}$ was found to be 0.9965 and the value of adj. $R_{c}{ }^{2}$ was found to be 0.9959 , indicating a good regression fit between the curves. Table 3 shows the values of the parameters $a, b, c$, and the coefficient of determination $R_{c}{ }^{2}$ at different $U / V$ ratios. For QMF operation

Table 3. Values of Parameters a, b, c, and CODs, for QMF Operation in Zone 3

\begin{tabular}{lcc}
\hline Variables & $U / V 104.81 \%$ & $U / V 104.82 \%$ \\
\hline $\mathrm{a}$ & 1662.19 & 2887.83 \\
$\mathrm{~b}$ & 3898.46 & 5100.54 \\
$\mathrm{c}$ & 0.9618 & 0.9773 \\
COD- $R_{c}{ }^{2}$ & 0.9993 & 0.9965 \\
Adj. $R_{c}{ }^{2}$ & 0.9990 & 0.9959 \\
\hline
\end{tabular}

in stability zone 3 (as for zone 1) it can be also be concluded that the predicted saturation behavior of resolution with increasing $N$ is a feature of the QMF.

\section{Conclusions}

Simulation results showing the performance of the QMF operating in stability zone 1 and zone 3 have been presented. The dependence of resolution $\mathrm{R}$ on number of $\mathrm{rf}$ cycles $\mathrm{N}$ experienced by the ions in the mass filter have been examined at different operating points. It has been observed that as the number of rf cycles increases, resolution increases with a finite slope and then the curve showing dependence starts to saturate. This is attributed to the fact that for a QMF there is a finite theoretical limit to its resolution for a particular value of operating point (scan line), since at that particular point, $\Delta q$ cannot go beyond a certain minimum value.

For stability zone 1 and for the linear region of the performance curve, QMF resolution has been found to be dependent on number of radio frequency cycles, according to $R=N^{n} / K$; however, for the saturated region of the curve (observed in practice and here modeled), QMF resolution has been found to be dependent on $N$ according to $R=q(1-$ $\left.2 c^{N}\right) / \Delta q$. Also, a relationship has been established between maximum resolution $R_{\max }$ and percentage $U / V$ ratio, given by $R_{\max }=q /(0.9330-0.00933 U / V)$.

For QMF operation in zone 3 the expression $R=a-b c^{N}$ simulates well the linear and saturated regions of the performance curve for a range of operational conditions, where $\mathrm{a}, \mathrm{b}$ and $\mathrm{c}$ are constants.

\section{Acknowledgments}

The authors thank Dr. Ken Evans for his guidance, advice, and constructive analysis during the course of this work.

\section{References}

1. Dawson, P.H.: Quadrupole Mass Spectrometry and Its Applications. Elsevier, Amsterdam (1976)

2. Taylor, S.; Srigengan, B.; Gibson, J.R.; Tindall, D.; Syms, R.; Tate, T.; Ahmad, M. A miniature mass spectrometer for chemical and biological sensing. Proc. SPIE 200 4036, 187-193 (2000)

3. Taylor, S., Tunstall, J.J., Leck, J.H., Tindall, R.F., Jullien, J.P., Batey, J., Syms, R.R.A., Tate, T., Ahmad, M.M.: Performance improvements for a miniature quadrupole with a micromachined mass filter. Vacuum 53, 203-206 (1999)

4. Brkic, B., France, N., Taylor, S.: Oil-in-water monitoring using membrane inlet mass spectrometry. Anal. Chem. 83, 6230-6236 (2011)

5. Paul, W., Raether, M.: Das elektrische massenfilter. Z. Phys. 40, 262$273(1955)$

6. Von Zahn, U.: Prazisions-massenbestimmungen mit dem elekrischen massenfilter. Z. Phys. 168, 129-142 (1962)

7. Brubaker, W.M., Tuul, J.: Performance studies of a quadrupole mass filter. Rev. Sci. Instrum. 35(8), 1007-1010 (1964)

8. Holme, A.E., Thatcher, W.J., Leck, J.H.: An investigation of the factors determining maximum resolution in a quadrupole mass spectrometer. $J$. Phys. E Sci. Instrum. 5, 429-433 (1972)

9. Dylla, H.F., Jarrell, J.A.: Transmission-resolution curves for a quadrupole mass spectrometer with separated $\mathrm{rf}$ and dc fields in the entrance aperture. Rev. Sci. Instrum. 47(3), 331-333 (1976) 
10. Brubaker, W.M.: An Improved Quadrupole Mass Analyzer. Adv. Mass Spectrom. 4, 293-299 (1968)

11. Batey, J.H.: Quadrupole gas analyzers. Vacuum 37, 659-668 (1987)

12. Muntean, F.: Transmission study for rf-only quadrupoles by computer simulations. Int. J. Mass Spectrom. Ion Process 151, 197-206 (1995)

13. Reuben, A.J., Smith, G.B., Moses, P., Vagov, A.V., Woods, M.D., Gordon, D.B., Munn, R.W.: Ion trajectories in exactly determined quadrupole fields. Int. J. Mass Spectrom. Ion Process 154, 43-59 (1996)

14. Gibson, J.R., Taylor, S., Leck, J.H.: Detailed simulation of mass spectra for quadrupole mass spectrometer systems. J. Vac. Sci. Technol. A 18 (1), 237-243 (2000)

15. Gibson, J.R., Taylor, S.: Prediction of quadrupole mass filter performance for hyperbolic and circular cross section electrodes. Rapid Commun. Mass Spectrom. 14, 1669-1673 (2000)
16. Du, Z., Douglas, D.J., Konenkov, N.: Elemental Analysis with Quadrupole Mass Filters Operated in Higher Stability Regions. J. Anal. At. Spectrom. 14(8), 1111-1119 (1999)

17. Hogan, T.J., Taylor, S.: Performance Simulation of a Quadrupole Mass Filter Operating in the First and Third Stability Zones. IEEE 57(3), 498-508 (2008)

18. March, R.E., Todd, J.F.J.: Quadrupole Ion trap Mass Spectrometry. Wiley, NJ (2005)

19. Douglas, D.J.: Linear quadrupoles in mass spectrometry. Mass Spectrom. Rev. 28, 937-960 (2009)

20. Zhang, S.C., Jin, J.M.: Computations of Special Functions. Wiley, New York (1996)

21. Konenkov, N.V., Kratenko, V.I.: Characteristics of a quadrupole mass filter in the separation mode of a few stability regions. Int. J. Mass Spectrom. Ion Process 108, 115-136 (1991) 